\title{
Tetanus: The First Child Case Report from Hevi Pediatric Teaching Hospital at Kurdistan Region - Iraq
}

\section{Federica Buzzi ${ }^{1}$, Nizar Bakir Yahya ${ }^{2}$, Khalaf Hussein Gargary ${ }^{3 *}$, Adel Hevan Al-Atroushy ${ }^{4}$ and Maiandi Stefano ${ }^{5}$}

${ }^{1} R N$, Head of Pediatric and Neonatal Intensive Care Clinical Training, E.U. Project

MADAD, Italian Association for Solidarity among Peoples, Duhok, KR, Iraq and

Nurse, Hematology and Bone Marrow Transplantation Unit and Pediatric

Immunohematology Unit, IRCCS San Raffaele Scientific Institute, Milan, Italy

${ }^{2}$ Assisntant Professor, Hevi Paediatric Teaching Hospital Duhok, KR, Iraq

${ }^{3}$ Professor, College of Medicine, University of Duhok, KR, Iraq

4Pediatrician FIBMS, Pediatric Intensive Care Unit, Hevi Pediatric Teaching

Hospital Duhok, KR, Iraq

${ }^{5} \mathrm{MsN}$, Professional Development and Research, Directorate of Health Professions,

ASST of Lodi, Italy

*Corresponding Author: Khalaf Hussein Gargary, Professor, College of Medicine, University of Duhok, KR, Iraq.

\section{Abstract}

Background: Tetanus is a vaccine-preventable disease caused by a robust neurotoxin produced by the spore-forming bacterium Clostridium tetani. However, numerous cases continue to be reported in internationally published literature indicating that tetanus continues to be a substantial problem despite the existence of vaccines.

In this case report, we describe the first case of tetanus at the Hevi Paediatric Teaching Hospital in Duhok, Iraq.

Case Presentation: Our patient was a 13-year-old boy admitted to the emergency room for widespread spasms and difficulty swallowing and urinating. The patient was admitted to the paediatric intensive care unit for suspicion of tetanus. The WHO international guidelines for the treatment and care of tetanus were adopted. After 17 days of hospitalization, the patient was discharged without neurological sequelae. Treatment for tetanus patients is long, complex, and multidisciplinary.

Conclusions: It is crucial to raise the awareness of tetanus and to vaccinate even the most vulnerable populations owing to the high costs of care and poor prognosis of patients with tetanus. In low- to middle-income countries such as Iraq, tetanus can impact overall healthcare management due to the complexity of patient care and high healthcare costs.

Keywords: Tetanus; WHO Guideline; Vaccine; Iraq; Kurdistan

\section{Abbreviations}

CPOT: Critical-Care Pain Observation Tool; TIG: Tetanus Immunoglobulin; WHO: World Health Organization

\section{Background}

Tetanus is a life-threatening disease caused by the spore-forming, obligate anaerobic bacterium Clostridium tetani [1]. Tetanus is an acute illness diagnosed clinically as a neuromuscular disorder characterized by increased muscle tone and spasms [2]. The condition further progresses to a painful, generalized spasm of the muscles in the neck, abdomen, or extremities and potential abdominal rigidity [3]. Disease severity is related to the speed of symptom evolution; more rapid progression is associated with more severe disease [4]. The incidence of tetanus has dramatically decreased 
worldwide since the mid- $20^{\text {th }}$ century, predominantly due to the development of the tetanus toxoid vaccine and the implementation of a vaccination programme [5]. Immunization and proper wound care are the best ways to prevent tetanus [6].

According to statistics from the World Health Organization (WHO) in 2019, 23 cases of tetanus occurred in Iraq among a total population of 39,310 people; however, tetanus vaccination is mandatory. For this reason, tetanus in children is a rare but still possible event.

\section{Case Presentation}

Our patient was a 13-year-old male refugee belonging to a poor and uneducated family from Sinjar (a rural area of Iraq). The patient arrived at the Emergency Department of the Hevi Paediatric Teaching Hospital in Duhok with generalized body spasms, dysphagia, and difficulty with movement and urination (Figure 1). He had a left foot ulcer with a necrotic base and purplish edges (Figure 2 ) resulting from the penetration of a nail 15 days earlier. The patient had not received a tetanus vaccine because the family refused the vaccine. During the physical examination, the patient was conscious but had difficulty speaking. He had rigours, tachycardia, elevated blood pressure and was tachypnoeic. His oxygen saturation was in the normal range. The patient also presented with severe chest pain. The description of all vital signs is shown in table 1. The chest X-ray and echocardiogram were normal. The complete blood count showed a white blood cell count of 11.5 cells $/ \mu$ l. The patient's serum sodium, potassium, and calcium levels were within normal ranges.

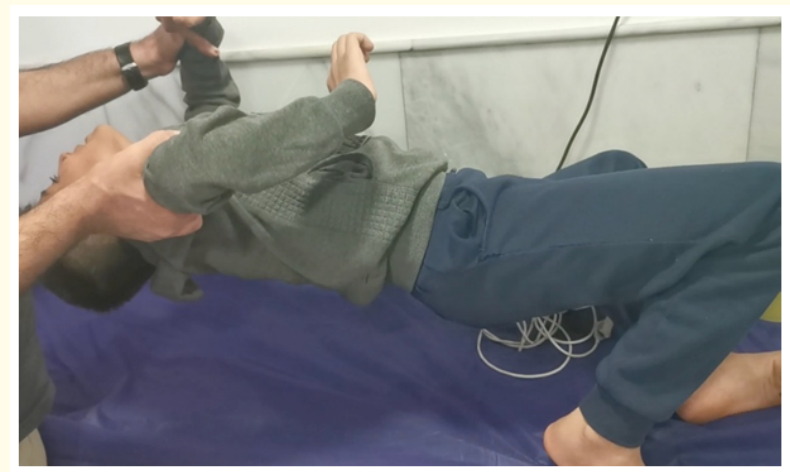

Figure 1: Involuntary upper extremity muscle spasms, followed by arching of the neck and back (opisthotonos) and generalized spasticity.

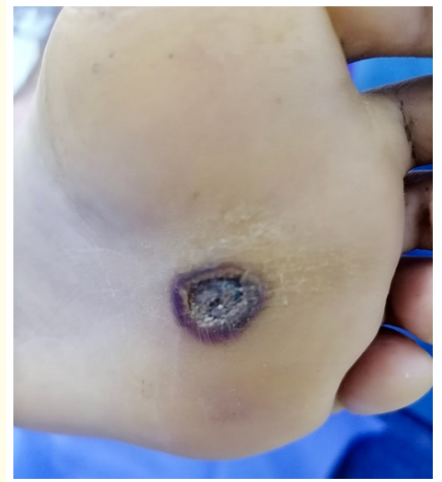

Figure 2: Foot injury before debridement.

\begin{tabular}{|l|c|c|c|}
\hline & $\begin{array}{c}\text { On arrival } \\
\text { time }\end{array}$ & $\begin{array}{c}\text { After 3 days } \\
\text { of admission }\end{array}$ & $\begin{array}{c}\text { After 10 days } \\
\text { of admission }\end{array}$ \\
\hline $\begin{array}{l}\text { Glasgow Coma Scale } \\
\text { (GCS) }\end{array}$ & 14 & 14 & 14 \\
\hline Temperature, ${ }^{\circ} \mathrm{C}$ & 36,5 & 38,9 & 36 \\
\hline Heart Rate, beats/min & 150 & 156 & 118 \\
\hline $\begin{array}{l}\text { Respiratory Rate, } \\
\text { breaths/min }\end{array}$ & 35 & 30 & 22 \\
\hline \begin{tabular}{l} 
Blood Pressure, $\mathrm{mmHg}$ \\
\hline Saturation, \%
\end{tabular} & $135 / 80$ & $140 / 90$ & $110 / 60$ \\
\hline $\begin{array}{l}\text { Critical-Care Pain } \\
\text { Observation Tool } \\
\text { (CPOT) }\end{array}$ & 7 & 93 & 99 \\
\hline $\begin{array}{l}\text { Comfortable Behavior } \\
\text { Scale }\end{array}$ & & 21 & 1 \\
\hline
\end{tabular}

Table 1: Table of the parameters and information on the patient in the different times detected.

The patient described here had an Ablett score of 3; therefore, severe trismus, generalized spasticity, prolonged spasms, severe dysphagia, and autonomic dysfunction presented as severe hypertension and tachycardia. Similar to most developing countries, tetanus is endemic in Iraq and remains a critical health problem, especially in rural areas. The patient was admitted to the paediatric intensive care unit for a preliminary diagnosis of tetanus based on his clinical history and the presence of the typical clinical manifestations of tetanus. Treatment commenced with the administration of 300 units of tetanus Immunoglobulin (TIG) via intramuscular in- 
jection. In addition, TIG was applied to the area around the wound after the cleaning and debridement of the necrotic tissue.

Penicillin G and metronidazole were concurrently started, and this treatment lasted for 14 days. Five hours after admission, the patient began to be irritable, with photophobia and intense pain and discomfort. The Behaviour Scale score was 26 , and the CriticalCare Pain Observation Tool (CPOT) score was 8. Therefore, the patient was transferred to the isolation room of the PICU. The initiation of pain-reducing (paracetamol), sedative (benzodiazepines), skeletal muscle relaxant, and antispasmodic (GABA derivative) therapy followed.

A nasogastric tube was inserted because the patient could not eat, and a catheter was inserted since the patient could not voluntarily urinate.

The patient was conscious after three days, and although there was an improvement in pain and discomfort, he was pyrectic, tachycardic, tachypnoeic, and hypertensive. Ten days of clindamycin treatment was started along with the previous antibiotic therapies because the foot injury swab was positive for Enterobacter cloacae.

The patient improved with time: his cardiovascular and respiratory parameters returned to normal levels, he was not irritated, and his pain was well controlled. The nasogastric tube and the bladder catheter were removed following recovery. After 17 days in intensive care, the patient was discharged. At the one-month follow-up visit, the patient was in good condition and without neurological, motor, or speech sequelae.

\section{Discussion and Conclusions}

The Ablett score is the most commonly used tool to determine the severity of tetanus. The reason for tetanus endemicity in Iraq could be that most cases occur in poor and illiterate populations with limited access to health service information and essential care $[7,8]$. Tetanus has a gradual onset of approximately 3 to 21 days after the infection, with symptoms typically worsening over a week. Symptoms start with spasms of the masseter muscle, resulting in trismus and lockjaw [9]. Targeted treatment should be instituted at the time of tetanus diagnosis to control and treat the disease [10]. Principles of the management of tetanus include admission to a dark and quiet room, muscle spasm and rigidity control, autonomic dysfunction control, ventilator support when needed, the neutral- ization of tetanus toxin, wound management, antibiotic administration, and the prevention of recurrence with booster vaccination. Benzodiazepines are still the cornerstone for sedation and spasm control. In children, tetanus vaccination, booster doses, and proper wound management are tetanus prevention strategies [11,12]. To treat this patient admitted to the Hevi Paediatric Teaching Hospital, all the indications described above were present, and the WHO guidelines for the administration of immunotherapy, antibiotic therapy, and the control of muscle spasms were adapted [13].

It is of paramount importance that patients with tetanus are recognized early and hospitalized for haemodynamic monitoring. These patients may develop blood pressure instability, arrhythmia, temperature instability, and rhabdomyolysis [14]. Central nervous system involvement can also cause intestinal disorders and bladder dysfunction and increase respiratory secretions, complicating disease management. Mortality is higher in very young than in elderly tetanus patients. The consequences of hypoxic brain injury include cerebral palsy, impaired mental abilities, and behavioural difficulties [15]. Several studies have found that the mortality of tetanus patients is approximately $40-80 \%$ (depending on severity) in low- and middle-income countries [16]. The most common cause of mortality is cardiovascular complications (40\%) and respiratory distress (15\%) [17]. Given the extensive hospital stay and the difficulty in treating tetanus patients, vaccination is considered one of the most appropriate planned public health interventions.

\section{Ethics Statement and Informed Consent}

This case report was developed in accordance with international ethical principles and respect for the confidentiality and anonymity of the participant's data was guaranteed. Consent to use the data collected for the purposes of the research study was requested in writing from the Duhok Directorate General of Health. The caregiver provided written informed consent to post case details and associated images.

Consent for the use of data collected for research purposes has been requested in writing from the Directorate General of Health in Duhok (Protocol n. 030322021-2-9/March 2021).

\section{Competing Interests}

The authors declare that they have no competing interests. 


\section{Funding}

The authors declare that they have not received funding.

\section{Author's Contributions}

Federica Buzzi and Hevan Adel Al-Atroushy conceptualized and designed the case report, collected the data and drafted the initial version of the manuscript.

Khalaf Hussein Gargary, Stefano Maiandi and Nizar Bakir Yahya have made a substantial contribution to the conception and design and have critically revised it for important intellectual contents. All authors approved the final manuscript assubmitted and agree to be accountable for all aspects of the work.

\section{Bibliography}

1. Rhinesmith E and Fu L. "Tetanus Disease, Treatment, Management". Pediatric Review 39.8 (2018): 430-432.

2. Woldeamanuel YW. "Tetanus in Ethiopia: unveiling the blight of an entirely vaccine-preventable disease". Current Neurology and Neuroscience Reports 12.6 (2012): 655-665.

3. Aggarwal M., et al. "Tetanus in pediatric patients--predictors affecting mortality and role of immunoglobulin". Indian Pediatrics 48.12 (2011): 987-988.

4. Ibinda F., et al. "Incidence and risk factors for neonatal tetanus in admissions to Kilifi County Hospital, Kenya". PLoS One 10.4 (2015): e0122606.

5. Lafta R and Hussain A. "Trend of vaccine preventable diseases in Iraq in time of conflict". Pan African Medical Journal 31 (2018): 130.

6. Scobie HM., et al. "Tetanus Immunity Gaps in Children 5-14 Years and Men $\geq 15$ Years of Age Revealed by Integrated Disease Serosurveillance in Kenya, Tanzania, and Mozambique". American Society of Tropical Medicine and Hygiene 96.2 (2017): 415-420.

7. Thwaites CL., et al. "Maternal and neonatal tetanus". Lancet (London, England) 385.9965 (2015): 362-370.

8. Yen LM and Thwaites CL. "Tetanus". Lancet (London, England) 393.10181 (2019): 1657-1668.

9. Oyedeji OA., et al. "Trends in neonatal and post-neonatal tetanus admissions at a Nigerian teaching hospital". Journal of Infection in Developing Countries 2012;6 (12): 847-853.
10. Ergonul O., et al. "An unexpected tetanus case". Lancet Infectious Disease 16.6 (2016): 746-752.

11. Rodrigo C., et al. "Pharmacological management of tetanus: an evidence-based review". Critical Care 18.2 (2014): 217.

12. Kyu HH., et al. "Mortality from tetanus between 1990 and 2015: findings from the global burden of disease study 2015". BMC Public Health 17.1 (2017): 179.

13. Tetanus vaccines: WHO position paper - February 2017". Releve Epidemiologique 92.6 (2017): 53-76.

14. Animasahun BA., et al. "Profile and outcome of patients with post-neonatal tetanus in a tertiary centre in south west $\mathrm{Ni}$ geria: any remarkable reduction in the scourge?" Pan African Medical Journal 21 (2015): 254.

15. Liu L., et al. "Global, regional, and national causes of under-5 mortality in 2000-15: an updated systematic analysis with implications for the Sustainable Development Goals". Lancet (London, England) 388.10063 (2016): 3027-3035.

16. Narang M., et al. "Epidemiological trends of tetanus from East Delhi, India: a hospital-based study". Journal of Infection and Public Health 7.2 (2014): 121-124.

17. Şık G., et al. "Intrathecal baclofen use in the management of tetanus related spasm: A case report". Turkish Journal of Pediatrics 61.1 (2019): 126-129.

\section{Assets from publication with us}

- Prompt Acknowledgement after receiving the article

- Thorough Double blinded peer review

- Rapid Publication

- Issue of Publication Certificate

- High visibility of your Published work

Website: www.actascientific.com/

Submit Article: www.actascientific.com/submission.php

Email us: editor@actascientific.com

Contact us: +919182824667 